\title{
Psyche und Krebs
}

n Deutschland erkranken jährlich über 340000 Personen an Krebs, wobei die Häufigkeit geschlechterspezifisch variiert. Bei den Frauen ist die Brustkrebserkrankung mit jährlich ca. 46000 Neuerkrankungen die häufigste Tumorerkrankung, während bei Männern das Lungenkarzinom mit jährlich 28000 Neuerkrankungen am häufigsten auftritt. Wenngleich hinsichtlich der Sterblichkeit für einige Krebsarten ein Rückgang der Mortalitätsrate in den letzten Jahren festzustellen ist, sind die Tumorerkrankungen nach den Herz-/Kreislauferkrankungen insgesamt gesehen die zweithäufigste Todesursache. Durch die Verbesserung der Behandlungsmöglichkeiten konnten die Überlebensraten deutlich verbessert werden, gleichzeitig jedoch sind die körperlichen und psychosozialen Belastungen und Folgeprobleme für die Betroffenen deutlich angestiegen.

Das vorliegende Themenheft ist daher dem Schwerpunktthema Psychoonkologie gewidmet. Die Psychoonkologie - synonym wird auch oft der Begriff „psychosoziale Onkologie" verwendet - befasst sich mit den komplexen psychosozialen Aspekten in der Entstehung, Behandlung und im Verlauf einer Krebserkrankung. Hierbei ist die Psychoonkologie weniger als eine eigene Disziplin zu verstehen, sondern als ein mehrere Fachdisziplinen umfassendes Gebiet. Unverzichtbare Elemente sind hierbei die Interdisziplinarität unter Einbeziehung von Fachgebieten wie bspw. medizinische Psychologie, Psychiatrie, Psychosomatik, Soziologie u.ä. In der Praxis bedeutet dies, dass in der Regel im onkologischen Behandlungsteam verschiedene Berufsgruppen (Ärzte, Pflegepersonal, Psychologen, Sozialarbeiter, Kunsttherapeuten u.a.) zusammenarbeiten, wobei je nach Behandlungssetting (Akutbehandlung, Rehabilitation, Nachsorge) die Schwerpunkte der psychosozialen Betreuung unterschiedlich verteilt sind. Im Jahre 1988 wurde innerhalb der Deutschen Krebsgesellschaft eine eigene Arbeitsgemeinschaft Psychoonkologie (PSO) gegründet, die neben der bereits existierenden Deutschen Arbeitsgemeinschaft für Psychoonkologie (dapo) die Psychoonkologie als Fachgesellschaften repräsentieren. Beide Gesellschaften verfolgen das Ziel der wissenschaftlichen Erforschung psychosozialer Aspekte der Krebserkrankung sowie deren Umsetzung und Integration in die Behandlung und Patientenbetreuung.

Trotz heute immer noch gravierender Mängel in der psychoonkologischen Versorgungsstruktur konnten erste psychosoziale Betreuungsstrukturen in Akutbehandlung, stationärer Rehabilitation und ambulanter Nachsorge aufgebaut werden. Wissenschaftliche Fortschritte wurden vor allem in der Erforschung der Krankheitsverarbeitung, der Entwicklung, Erprobung und Evaluation spezieller Interventionen für Krebspatienten sowie der Methodenentwicklung im Bereich der Lebensqualitätsforschung erreicht. Die wissenschaftliche Produktivität lässt sich neben den zahlreichen Arbeiten in medizinpsychologischen oder psychosomatischen Fachzeit- schriften auch in der Gründung spezieller Fachzeitschriften wie bspw. "Journal of Psychosocial Oncology“, „Psycho-Oncology“ oder „Quality of Life Research“ ablesen. Das erste Handbuch der Psychoonkologie liegt mittlerweile in einer vollständig neubearbeiteten Form vor (Holland, 1998) und gibt einen umfassenden Überblick über das breite Spektrum psychoonkologischer Forschung. Ebenso konnten in Deutschland nationale Förderprogramme zur Forschungsförderung den im internationalen Vergleich zu verzeichnenden Rückstand psychoonkologischer Forschung deutlich verbessern helfen.

Das wachsende Interesse an der Psychoonkologie macht deutlich, dass in der modernen Onkologie mit ihrer Spezialisierung und Hochtechnisierung die Patienten nur dann gut behandeln werden können, wenn neben den körperlichen Aspekten auch ihre psychosozialen und geistigen Bedürfnisse wahrgenommen werden und in der Behandlung Berücksichtigung finden. Auch seitens der Patienten wird dies zunehmend eingefordert: Patienten wollen angemessen informiert und aufgeklärt und in medizinische Entscheidungen miteinbezogen werden. Sie nehmen stärker als bisher eine aktive Rolle ein und versuchen ihre eigenen Rechte zu vertreten. In dieser Entwicklung können psychoonkologische Forschungsergebnisse viel dazu beitragen, Behandlungsund Versorgungsstrukturen zu verbessern. Dazu muss die Psychoonkologie zukünftig ihre Erkenntnisse und ihr Wissen stärker auch in versorgungs- und gesundheitspolitische Entscheidungen einbringen. Die Ausarbeitung von Leitlinien und Behandlungsstandards für die Psychoonkologie sind ein wichtiger Schritt in diese Richtung.

Für das vorliegende Themenheft wurden einige zentrale Schwerpunkte aus dem breiten Themenspektrum der Psychoonkologie ausgewählt, die exemplarisch das Gebiet in seinen wesentlichen Grundlagen und Forschungsstrategien vorstellen. Reinhold Schwarz führt den aktuellen Kenntnisstand im Bereich der Psychoätiologie der Krebserkrankung aus. Der Beitrag von Monika Keller gibt einen Überblick über das Thema der psychoonkologischen Interventionen. Die Auswirkungen einer Krebserkrankung auf die Familie und das soziale Umfeld werden in dem Beitrag von Werner Geigges dargelegt. Schließlich wird das Thema Lebensqualität am Beispiel des in den letzten Jahren zunehmend in den Mittelpunkt gerückten Problembereichs der tumorbedingten Fatigue von Ulrich Rüffer vorgestellt.

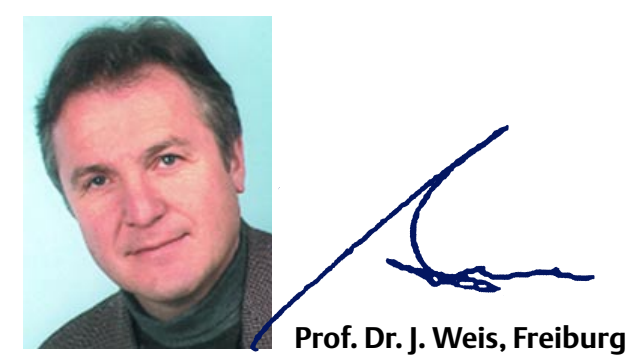

